\title{
A formação acadêmica do enfermeiro e os princípios organizacionais do sus no âmbito da saúde integral a população negra
}

\author{
Nursing academic training and SUS organizational principles in the framework of black \\ population
}
Entrenamiento académico de enfermería y principios organizativos de SUS en el marco de la población negra

Alda Lima Lemos ${ }^{1 *}$, Edna Ferreira Galvão.

\begin{abstract}
RESUMO
Objetivo: Evidenciar a formação acadêmica do enfermeiro e os princípios organizacionais do Sistema Único de Saúde (SUS) que contribuem para uma formação direcionada ao atendimento da população negra, refletindo sobre a Política Nacional de Saúde Integral da População Negra. Métodos: Trata-se de uma revisão sistemática de literatura, realizada nos bancos de dados: Scientific Electronic Library Online, National Library of Medicine, Biblioteca Virtual em saúde e Portal de Periódicos da Coordenação de Aperfeiçoamento de Pessoal de Nível Superior. Os artigos abordaram a formação acadêmica de enfermagem e os princípios do SUS, com ênfase na saúde da população negra. Foram encontrados 579 artigos, 310 foram excluídos pelo título, 280 pelo resumo, 12 por duplicidade e 15 por não se enquadrarem nos objetivos, constituindo 3 artigos nesta revisão. Revisão Bibliográfica: Os estudos evidenciam que a formação do enfermeiro é orientada pelas diretrizes curriculares de enfermagem, norteada pelos princípios organizativos do SUS, contudo apresenta dificuldades e fragilidades que devem ser repensadas como a interdisciplinaridade e transversalidades dos conteúdos. Conclusão: A formação dos enfermeiros precisa estar direcionada para atender às necessidades sociais de saúde, sendo orientada pelas Diretrizes Curriculares de Enfermagem e em consonância com os princípios do SUS, abordando a saúde da população negra.
\end{abstract}

Palavras-chave: Sistema único de saúde, Enfermeiras e enfermeiros, Capacitação profissional.

\begin{abstract}
Objective: To highlight the academic training of nurses and the organizational principles of the Unified Health System (SUS) that contribute to training aimed at serving the black population, reflecting on the National Policy for Integral Health of the Black Population. Methods: This is a systematic literature review performed in the following databases: Scientific Electronic Library Online, National Library of Medicine, Virtual Health Library and Periodical Portal of the Coordination for the Improvement of Higher Education Personnel. The articles addressed academic nursing education and the principles of SUS, with an emphasis on the health of the black population. 579 articles were found, 310 were excluded by title, 280 by abstract, 12 by duplicity and 15 because they did not fit the objectives, constituting 3 articles in this review. Bibliographic Review: Studies show that the training of nurses is guided by the curricular guidelines of nursing, guided by the organizational principles of SUS, however it presents difficulties and weaknesses that must be rethought as the interdisciplinary and transversalities of the contents. Conclusion: The training of nurses needs to be directed to meet social health needs, being guided by the Nursing Curriculum Guidelines and in line with SUS principles, addressing the health of the black population.
\end{abstract}

Keywords: Unified health system, Nurses, Professional training.

1 Universidade do Estado do Pará, (UEPA) Santarém-PA. *E-mail: lemos.alda@bol.com.br 


\section{RESUMEN}

Objetivo: Destacar la educación académica de las enfermeras y los principios organizativos del Sistema Único de Salud (SUS) que contribuyen a una capacitación dirigida a la población negra, reflexionando sobre la Política Nacional de Salud integral de la población negra. Métodos: Es una revisión sistemática de la literatura, realizada en las siguientes bases de datos: Biblioteca Electrónica Científica en línea, Biblioteca Nacional de Medicina, Biblioteca Virtual en Salud y Portal Periódico de la Coordinación para la Mejora del Personal de Educación Superior. Los artículos abordaron la educación académica en enfermería y los principios del SUS, con énfasis en la salud de la población negra. Se encontraron 579 artículos, 310 fueron excluidos por título, 280 por resumen, 12 debido a duplicidad y 15 porque no se ajustaban a los objetivos, constituyendo 3 artículos en esta revisión. Revisión bibliográfica: Los estudios demuestran que la formación de enfermeras se guía por las pautas curriculares de enfermería, guiadas por los principios organizativos del SUS, sin embargo, presenta dificultades y debilidades que deben ser repensados como la interdisciplinariedad y transversalidades de los contenidos. Conclusión: La capacitación de las enfermeras debe estar dirigida a satisfacer las necesidades sociales de salud, guiándose por las Pautas del plan de estudios de enfermería y en línea con los principios del SUS, abordando la salud de la población negra.

Palabras clave: Sistema único de salud, Enfermeras y enfermeros, Capacitación profesional.

\section{INTRODUÇÃO}

Os princípios organizativos do sistema único de saúde (SUS), no âmbito da saúde integral da população negra, constitui-se como uma política pública, que contribui para a redução das desigualdades e vulnerabilidades sociais e de saúde. A redução das vulnerabilidades e desigualdades na população negra tem ocorrido por uma cadeia de políticas de promoção e prevenção, desenvolvidas pelo Ministério da Saúde, dentre elas está a Política Nacional de Saúde Integral da População Negra (PNSIPN), que é uma política pública de promoção e prevenção da saúde da população negra, a qual garante aos usuários do Sistema Único de Saúde (SUS) o princípio da equidade na positivação do direito humano à saúde desta população, em suas características de promoção, prevenção, atenção, tratamento e recuperação de doenças e agravo transmissíveis e não transmissíveis (CHEHUEN NETO JA, et al., 2015).

A Política Nacional de Saúde Integral da População Negra (PNSIPN), instituída pela Portaria do Ministério da Saúde/Gabinete do Ministro no 992 de 13 de maio de 2009, busca garantir a equidade e a efetivação do direito à saúde aos afro-brasileiros. Tendo como marco o reconhecimento do racismo, das desigualdades étnico-raciais e do racismo institucional como determinantes sociais das condições de saúde, estabelecendo diretrizes, estratégias e responsabilidades da gestão em todas as esferas de governo (BRASIL, 2009).

É uma medida compensatória na área de saúde, na tentativa de minimizar os efeitos da discriminação e da exploração sofridas pelos negros ao longo da história brasileira. Destaca-se nesta política, um dos princípios do SUS, a equidade que aproxima o profissional enfermeiro da realidade local de saúde, diagnosticando a situação de saúde, as vulnerabilidades e desigualdades sociais, que poderão comprometer o acesso aos serviços de saúde das populações negras (BRASIL, 2017).

O censo realizado pelo Instituto Brasileiro de Geografia e Estatística (IBGE) de 2010, demostrou que a quantidade de pessoas que se autodeclaram pardas ou negras foi de $43,1 \%$ e de $7,8 \%$, respectivamente. Portanto, perfazendo mais de 50,7\% da população brasileira (CHEHUEN NETO JA, et al., 2015).

Apesar desta realidade desafiadora, verifica-se um panorama de desigualdade de acesso, assistência e tratamento, nos espaços públicos de saúde, provocada pela discriminação e/ou preconceitos nos serviços públicos, de saúde, afetando negativamente a saúde da população negra brasileira e dificultando a efetivação de uma assistência de saúde integral (MENDES VS, et al., 2015).

No campo da saúde da população negra, considera-se que a morbimortalidade no Brasil, tem cor, que as formas de adoecer e morrer desse grupo social, está relacionada às condições materiais e sociais (LAGES 
SRC, et al., 2017). As dificuldades de acesso as políticas públicas de saúde, as condições insalubres de vida, dificuldade de trabalho e renda e os comportamentos racistas e de exclusão social, e a negação da cidadania, contribuem para provocar iniquidades e agravos na saúde dos negros (MATOS CC e TOURINHO FS, 2018).

Neste contexto, a saúde do negro ficou relegada a uma situação de vulnerabilidade social, econômica, vivendo em condições indignas de habitação, precariedade na alimentação e de dificuldade de obter os tratamentos corretos para doenças que mais os acometiam, em virtude desse quadro. Os negros apresentavam maiores índice de doenças infectocontagiosas, parasitárias, doenças transmitidas por vetores, doenças geneticamente herdadas e outros quadros de adoecimento provocados por maus tratos e violências. Esta realidade começou a mudar com o movimento da reforma sanitária, iniciada em 1970, com ideais de mudanças e transformações necessárias na saúde (PORTO A, 2006).

Diante desse quadro, a formação do profissional de saúde, necessita acompanhar as mudanças impostas à sociedade, devendo estar articuladas às demandas de saúde, sociais e culturais da população, bem como, gerar egressos sensibilizados e comprometidos com a saúde e com a capacidade técnica, e humanística para atuar em consonância com os modelos assistenciais preconizados nos serviços, ofertando ações e serviços com resolutividade dos problemas através dos princípios organizativos do SUS (MAKUCH DMV e ZAGONELI IPS, 2017).

Conforme a Resolução do Conselho Nacional de Educação/Câmara de Educação Superior (CNE/CES) no 3, de 7 de novembro de 2001; as diretrizes curriculares do curso de graduação em enfermagem, estabelece o perfil do formando egresso/profissional com uma formação generalista, humanista, crítica e reflexiva; qualificado para o exercício de Enfermagem, no rigor científico e intelectual e pautado em princípios éticos; capaz de conhecer e intervir sobre os problemas/situações de saúde-doença, mais prevalentes no perfil epidemiológico nacional, com ênfase na sua região de atuação, identificando as dimensões biopsicossociais dos seus determinantes (BRASIL, 2001).

No estudo de Santana RAR, et al. (2019), observou-se que a abordagem da saúde integral da população negra ainda encontra dificuldade para se consolidar nos currículos de cursos da saúde, apesar de presente nas instituições de ensino, ainda se apresenta com pouca visibilidade, vista de forma obrigatória e impositora por parte do Ministério de saúde e não reconhecida sua importância e debate para a saúde.

Diante das novas configurações do mundo globalizado e da modernização científica e tecnológica e a constante rapidez das velocidades das informações no mundo contemporâneo. Há necessidade de romper com modelos atuais de formação dos profissionais de saúde, propondo mudanças inovadoras no que tange os currículos de formação em saúde, com intuito de capacitar o futuro enfermeiro egresso comprometido com a vigilância em saúde com capacidade de compreender e recompreender os determinantes sociais de saúde, com propósito de inserir os saberes tecnológicos apreendidos durante a formação acadêmica para atuar no desenvolvimento de práticas cuidado em saúde; articulando os conhecimentos empíricos com os científico, associando teoria com a prática. Procura-se entender as crenças e valores culturais da população dentro de uma visão ampliada de como obter a saúde no enfoque da integralidade, buscando gerar vínculos tanto individuais como coletivos, com o propósito de atender as necessidades de saúde da população (PIRES AS, et al., 2014).

As Instituições de Ensino Superior (IES) são uma estrutura organizacional de grande relevância para o desenvolvimento da sociedade, são consideradas como organismo social que tem como responsabilidade a formação e capacitação de profissionais e tem um papel de qualificar e conscientizar os cidadãos do seu papel social.

Possui um papel fundamental na formação, pois estão em constante interação com a sociedade, sendo responsáveis pela formação da cidadania, política, cultural e social de seus egressos. Devem estar organizadas sistematicamente pelas diretrizes e base de educação nacional e que tem autonomia de elaborar seus projetos pedagógicos curriculares de ensino, tendo como bases as orientações das diretrizes curriculares nacionais de cada curso, provendo meios e estratégias pedagógicas de ensino e aprendizagem para formação de profissionais capacitados e habilitados (GAZZONI F, et al., 2017) 
Em relação ao ensino direcionado para formar os enfermeiros, nas propostas curriculares é que os egressos tenham recebido um aprendizado direcionado para serem protagonistas nas ações e serviços do SUS de acordo com as especificidades epidemiológica de cada região, e com uma formação mais próxima da realidade local, para atuar no mercado de trabalho e que sejam profissionais que possam adequar-se as diversidades dos cenários de saúde (GANASSIN FMH, 2015).

Os projetos políticos pedagógicos de enfermagem devem ser elaborados para que os currículos sejam construídos de forma que os egressos possam ter uma visão integradora, polissêmica, multifacetadas e inovadora, capacitando o estudante desenvolver suas funções profissionais com competência, habilidade e atitude ética. É indicado que seja construído de forma coletiva, em um movimento consciente de cooperação e solidariedade. Partindo desta premissa é oportuno compreender a percepção dos professores que participaram do referido processo de construção (MAGALHÃES SMF, et al.,2017).

A elaboração do projeto político pedagógico exige uma profunda reflexão sobre as finalidades da instituição, seu papel social e a definição clara do caminho a ser percorrido e da operacionalização das ações que serão empreendidas. Tendo expressos as prioridades na formação do cidadão, as atividades pedagógicas e didáticas que levam a instituição a alcançar os objetivos.

Alinhando-se as adaptações culturais; a exemplo das questões raciais respeitando a diversidade ética, introduzindo práticas não discriminatórias e impulsionando o egresso para que possa ressignificar o seu papel de agente social de cidadania, desenvolvendo uma liderança responsável (MAGALHÃES SMF, et al.,2017).

Nessa perspectiva, Inocêncio JF e Tavares MM, (2014) afirmaram que os profissionais docentes enfermeiros mantém conviç̧ões distorcidas sobre raça e saúde. Suas concepções baseiam-se em dimensões biológicas, tematizando pacientes negros como potenciais patogênicos a certos tipos de doenças, sem reconhecimento do racismo como um dos influenciadores desse processo.

Há necessidade de que as universidades possam adequar-se aos novos modelos de atenção à saúde da atualidade, sendo desafiadas no sentido de reorientar o processo pedagógico na formação profissional dos enfermeiros, enfatizando a relevância das políticas públicas de saúde no enfoque transversal e interdisciplinar, direcionada a este grupo populacional (SANTOS RG e TOCANTINS FR, 2015).

Objetivou-se, com esta pesquisa evidenciar a formação acadêmica do enfermeiro e os princípios organizacionais do SUS que contribuem para uma formação direcionada a população negra, refletindo sobre a política nacional de saúde integral da população negra.

\section{MÉTODOS}

Para construção deste estudo foi realizada uma revisão sistemática em banco de dados que apresentaram publicações de artigos completos entre os anos de 2014 a 2019, nas bases de dados: Scientific Electronic Library Online (SCIELO), National Library of Medicine (Pubmed), Biblioteca Virtual em saúde (BVS) e Portal de Periódicos da Coordenação de Aperfeiçoamento de Pessoal de Nível Superior (CAPES).

Como suporte, utilizou-se o que está recomendado do método de Principais Itens para Relatar Revisões sistemáticas e Meta-análises (PRISMA), adaptado para esta revisão, que é composto por uma lista de 27 itens e um fluxograma que norteiam os autores a produção de melhores e confiáveis produções de revisões (MOHER D, et al., 2015).

O questionamento principal para construção deste artigo foi: Como a política nacional de Saúde Integral da população Negra tomam o corpo no processo de formação da enfermagem? Os princípios organizativos do SUS colaboram para uma assistência focada nesta política da saúde da população negra?

$\mathrm{Na}$ busca pelos artigos, utilizou-se os termos "formação", "enfermeiros" e "população negra" foram agrupados com o operador "AND", porém não foram encontrados nas bases de dados. Para combinar os termos, foi inserido o operador "OR" entre as palavras, em que os artigos encontrados continham pelo menos um dos termos de busca. 
Os critérios de inclusão utilizados foram: artigos originais de cunho nacional (Brasil) que abordassem a temática relacionada com a formação do enfermeiro, sobre a política de saúde integral da população negra, e os princípios organizativos do SUS. Foram utilizados apenas artigos científicos originais. E foram excluídos trabalhos de revisão, livros, capítulos de livros, relato de experiência e os editoriais, dissertações e teses, além de artigos que não tratassem especificamente da formação de enfermagem e dos cuidados em saúde com a população negra.

Os trabalhos selecionados, a partir dos termos, foram organizados em três etapas. Na $1^{\text {a }}$ etapa houve a leitura dos títulos encontrados nos bancos de dados Capes, Pubmed, BVSaúde e Scielo, com posterior inclusão e exclusão, seguindo os critérios no fluxograma (Figura 1).

$\mathrm{Na} 2^{\mathrm{a}}$ etapa, foram lidos os resumos para averiguação de quais estudos se enquadrariam na temática abordada e na $3^{\text {a }}$ etapa, leitura do texto na íntegra afim de fortalecer os itens de inclusão e exclusão para compor a amostra para a revisão.

Ainda como parte integrante do processo de seleção dos trabalhos para construir este artigo, todos que fossem repetidos nas diferentes bases de dados, foram excluídos logo após a leitura na íntegra, para evitar duplicidade. Vale ressaltar que todas as etapas foram realizadas por mais de um pesquisador, afim de dar maior confiabilidade e exatidão na busca e análise dos resultados.

Foram encontrados 579 artigos a partir da busca de dados usando os termos. Desse total, 120 foram encontrados na Capes, 344 na Pubmed, 06 na BVSaúde e 109 na Scielo. De acordo com os critérios de elegibilidade foram selecionados três artigos compreendendo entre os anos de 2014 a 2019 para fazer parte desta revisão (Figura 1).

Figura 1 - Fluxograma com o quantitativo de trabalhos encontrados e selecionados, após aplicação dos critérios estabelecidos.

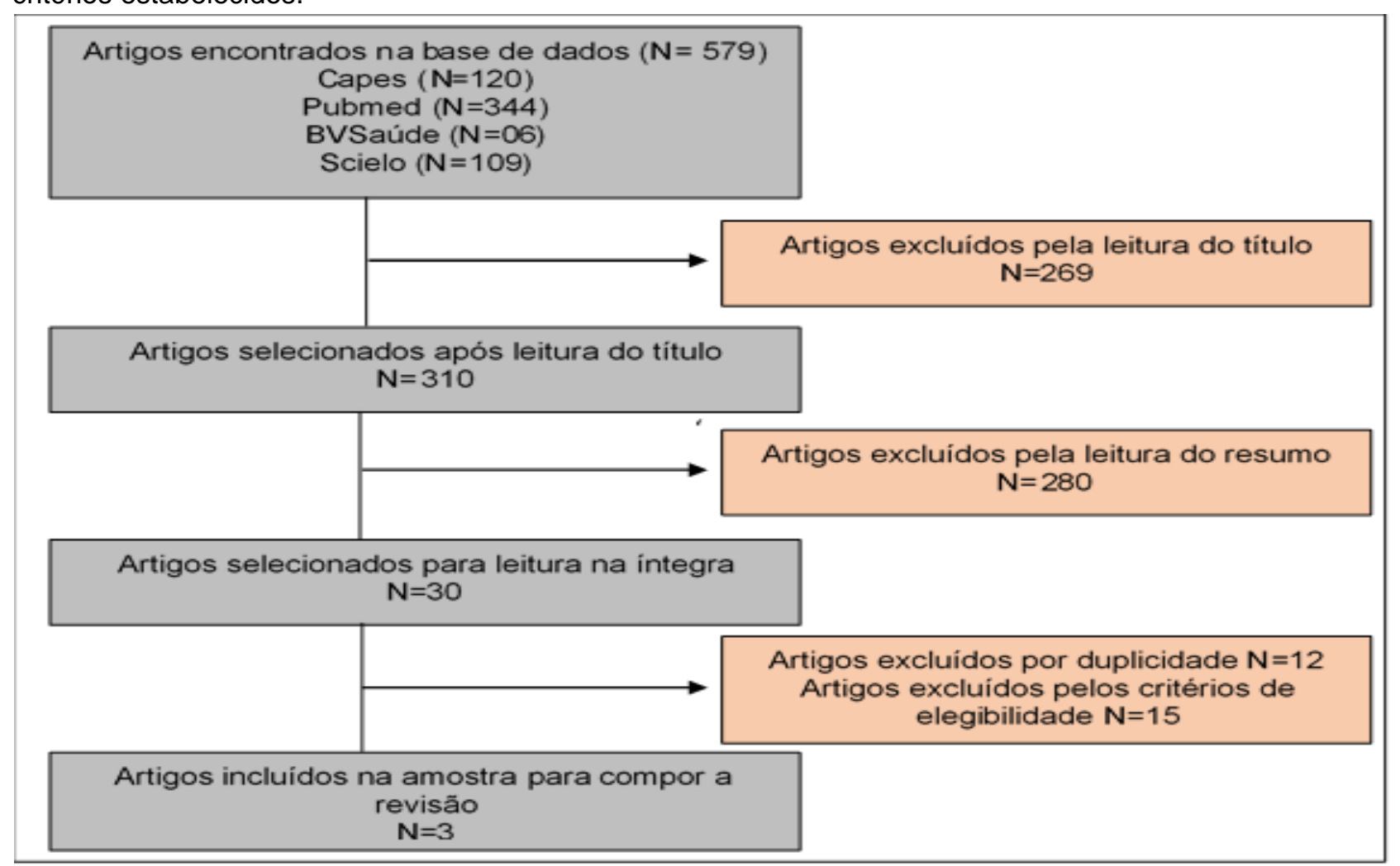

Fonte: Lemos AL e Galvão EF, 2020.

Os resultados foram organizados em quadros, evidenciando os autores, ano, objetivo principal, público alvo, método utilizado e resultados encontrados. 


\section{RESULTADOS E DISCUSSÃO}

Os artigos dos autores selecionados envolveram docentes e alunos com questionamentos sobre a formação acadêmica orientada nos princípios organizativos do sistema único de saúde, tendo em vista a constituição federal de 1988, que amplia os direitos de saúde. E sobre as diretrizes curriculares de enfermagem no enfoque contemporâneo, com uma visão diferenciada do processo saúde e doença, com integração de conteúdos interdisciplinares no processo de ensino. E também sobre o conhecimento de usuários negros sobre a existência da política direcionada a saúde da população negra. Ressaltando que não foram encontrados estudos específicos, que tratem sobre a atuação de enfermagem direcionada a saúde da população negra. 


\section{Revista Eletrônica Acervo Saúde / Electronic Journal Collection Health | ISSN 2178-2091}

Quadro 1 - Análise dos estudos encontrados nas bases de dados a partir dos critérios de inclusão e exclusão, entre os anos de 2014 e 2019 , N=3.

\begin{tabular}{|c|c|c|c|c|}
\hline $\begin{array}{c}\text { Autores/a } \\
\text { no }\end{array}$ & Objetivo & Amostra & Método & Resultados \\
\hline $\begin{array}{c}\text { WINTERS } \\
\text { JRF, et al., } \\
2016 .\end{array}$ & $\begin{array}{l}\text { (dentificar com os } \\
\text { formandos de graduação } \\
\text { de enfermagem de uma } \\
\text { universidade pública da } \\
\text { Região do Sul do Brasil, } \\
\text { percebem o seu processo } \\
\text { de formação em } \\
\text { consonância com os } \\
\text { princípios do SUS. }\end{array}$ & $\begin{array}{c}11 \text { estudantes } \\
\text { de enfermagem } \\
\text { com idade de } 21 \\
\text { a } 30 \text { anos. }\end{array}$ & $\begin{array}{l}\text { Estudo de caráter qualitativo, } \\
\text { descritivo e exploratório, por } \\
\text { meio de entrevistas com roteiro } \\
\text { semiestruturado, com analise de } \\
\text { conteúdo, que se organizou em } \\
\text { pré-análise, exploração do } \\
\text { material, tratamento dos dados } \\
\text { e inferência e interpretação. } \\
\text { Com a definição de categorias; } \\
\text { o processo de formação na } \\
\text { percepção dos formandos; e a } \\
\text { subcategoria; A formação está } \\
\text { direcionada a realidade e com } \\
\text { ênfase no SUS. }\end{array}$ & 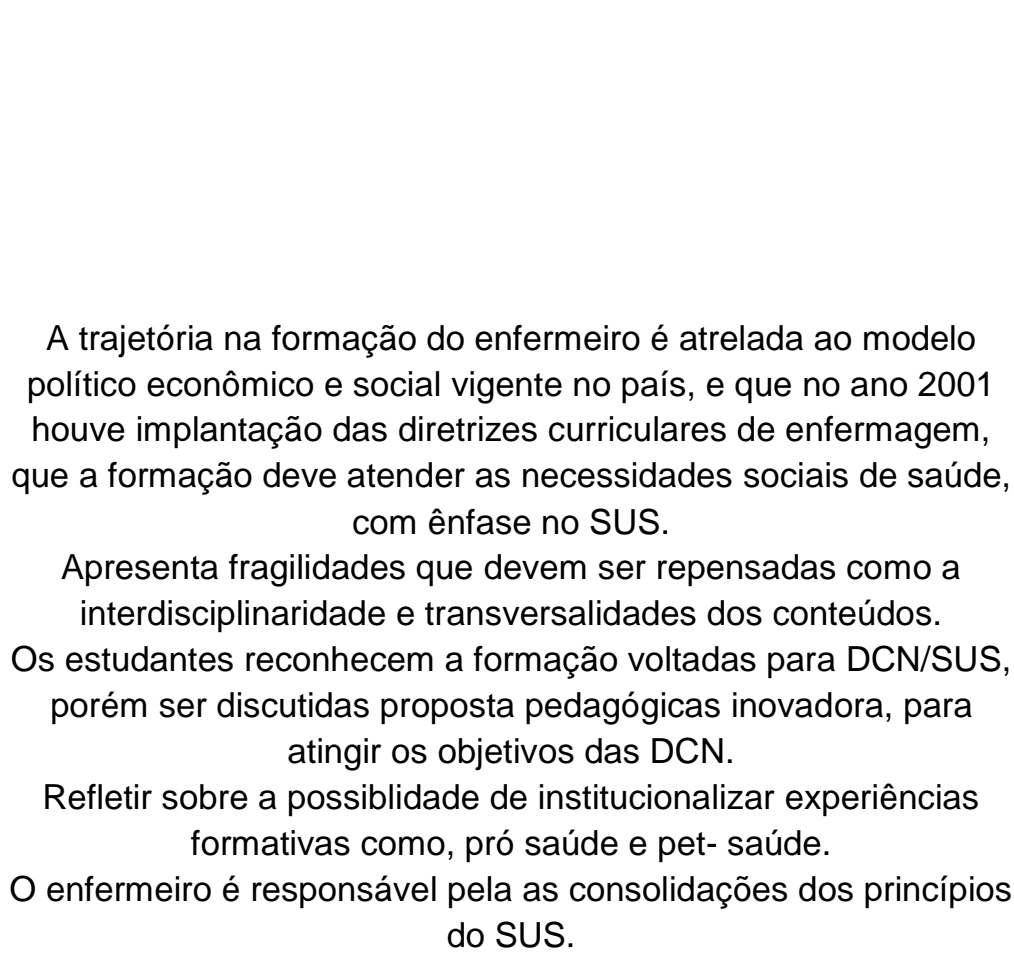 \\
\hline
\end{tabular}

REAS/EJCH | Vol.Sup.n.45 | e2943 | DOI: https://doi.org/10.25248/reas.e2943.2020 Página 7 de 11 


\begin{tabular}{|c|c|c|c|c|}
\hline $\begin{array}{l}\text { PERES } \\
\text { CRFB, et } \\
\text { al., 2018. }\end{array}$ & $\begin{array}{c}\text { Compreender } \\
\text { ascontradições } \\
\text { envolvidas no processo } \\
\text { de reconstrução curricular } \\
\text { a partir da ótica de } \\
\text { docentes de cursos de } \\
\text { instituições públicas e } \\
\text { privada. }\end{array}$ & $\begin{array}{l}21 \text { docentes de } \\
\text { instituições } \\
\text { pública e } \\
\text { privada com } \\
\text { cursos de } \\
\text { graduação de } \\
\text { enfermagem no } \\
\text { estado de São } \\
\text { Paulo. }\end{array}$ & $\begin{array}{l}\text { Pesquisa qualitativa na } \\
\text { modalidade interpretativa, } \\
\text { realizada por meio de } \\
\text { entrevistas com perguntas } \\
\text { semiestruturadas, com análise } \\
\text { pautada na hermenêutica- } \\
\text { dialética, tendo como referência } \\
\text { os princípios e diretrizes do SUS } \\
\text { e as diretrizes curriculares } \\
\text { nacionais de enfermagem. }\end{array}$ & $\begin{array}{l}\text { Constatou-se a necessidade de mudanças, mas permeiam } \\
\text { resistências, devido estarem acostumados com a fragmentação } \\
\text { dos conteúdos, disciplinas e a falta de diversificação dos cenários } \\
\text { de atenção, e que as mudanças ocorrerem de forma processual e } \\
\text { deverá ocorrer a incorporação de novos saberes. } \\
\text { Ter uma visão ampliada do processo de saúde-doença, } \\
\text { contrapondo-se a teorias biologicistas para os agravos à saúde, e } \\
\text { que o conceito de saúde não é universal e sim construído no } \\
\text { contexto histórico e social de cada época e sociedade refletindo } \\
\text { sua condição social, econômica, política e cultural. Apontam para } \\
\text { diversificar os cenários de ensino- aprendizagem, possibilitando } \\
\text { os estudantes vivenciar as realidades do cotidiano nos serviços } \\
\text { de saúde. Integração entre as disciplinas do ciclo básico e clínico } \\
\text { e a organização curricular. O docente deve se aproximar do } \\
\text { campo, para ter a percepção das necessidades de saúde. Para } \\
\text { atender às orientações das políticas de formação em saúde o } \\
\text { estudo pode mobilizar reflexões e possibilidade a compreensão } \\
\text { do movimento dialético, inerente ao processo de mudanças. }\end{array}$ \\
\hline $\begin{array}{l}\text { CHEHUEN } \\
\text { NETO JA, } \\
\text { et al., } \\
2015 .\end{array}$ & $\begin{array}{l}\text { Investigar o conhecimento } \\
\text { da população negra } \\
\text { acerca da política, seus } \\
\text { potenciais benefícios e as } \\
\text { dificuldades de acesso à } \\
\text { saúde. }\end{array}$ & $\begin{array}{l}391 \text { indivíduos } \\
\text { de cor negra e } \\
\text { ser morador de } \\
\text { Juiz de } \\
\text { Fora/Minas } \\
\text { Gerais. }\end{array}$ & $\begin{array}{l}\text { O delineamento do estudo é do } \\
\text { tipo transversal, exploratório e } \\
\text { descritivo com característica } \\
\text { quantitativa, com aplicação de } \\
\text { questionário estruturado por } 6 \\
\text { questões de cunho } \\
\text { socioeconômico e } 16 \text { questões } \\
\text { dicotômicas, com análise } \\
\text { descritiva e exploratória e teste } \\
\text { do qui-quadrado. }\end{array}$ & $\begin{array}{l}\text { 90,5\% relataram desconhecer a política de saúde da população } \\
\text { negra. } \\
60,9 \% \text { concordam que há necessidade da política. } 52,7 \% \text { declara } \\
\text { a PNSIPN, tende a reforçar a discriminação racial. } 56,4 \% \\
\text { relataram o acesso difícil aos serviços de saúde. } 58,8 \% \text { a } \\
\text { população negra, tem menos preocupação com a saúde. }\end{array}$ \\
\hline
\end{tabular}

REAS/EJCH | Vol.Sup.n.45 | e2943 | DOI: https://doi.org/10.25248/reas.e2943.2020 Página 8 de 11 
Para a formação do egresso de enfermagem em um profissional é necessário ter competência técnica, política e humanística, no mundo contemporâneo para atuar com efetividade no campo profissional. Portanto, as instituições de ensino devem ser encorajadas a desenvolver o acadêmico para que seja dotado de habilidade, conhecimento, raciocínio, percepção e sensibilidade para as questões da vida e da sociedade, tendo capacidade para intervir em ambientes complexos de saúde (WINTERS JRF, et al., 2016).

No mundo contemporâneo, a profissionalização do profissional enfermeiro, deverá formar um egresso com competência técnica, política e humanística. As instituições de ensino, são encorajados no sentido que o egresso seja

Portanto, esta formação deve estar direcionada para atender as necessidades sociais de saúde, orientada pela as diretrizes curriculares de enfermagem e em consonância com os princípios e diretrizes do SUS, assegurando a integralidade do cuidado, acesso aos serviços com qualidade técnica e humanização nos contextos de saúde (PERES CRFB, et al., 2018).

Devendo haver reflexões nos processos educacionais com abordagem inovadora, sobre os conceitos do processo saúde e doença, para além da cura numa proposta de uma visão ampliada do conceito de saúde, contrapondo-se a visão biologicista, que ainda perduram no processo de ensino (PERES CRFB, et al., 2018).

O estudo dos autores Winters JRF, et al. (2016) discutem que o modelo de saúde, antes da reforma sanitária era fragmentado e departamentalizado, excluía a população carente, com maiores vulnerabilidades sociais e econômicas, concentrando a atenção a saúde em consultórios médicos e hospitais privados, privilegiando a doença. Diante das desigualdades sociais, os princípios do SUS devem fundamentar a formação dos profissionais de enfermagem.

No entanto, é necessário compreender o movimento dialético para as novas concepções pedagógicas, direcionadas para uma formação qualitativa do enfermeiro no mercado de trabalho, apresentando obstáculos tais como a dificuldade dos docentes em compreender o real significado do processo, pois, muitos resistem a essas mudanças. Por outro lado, não há como permanecer em uma abordagem pedagógica que não atende as necessidades da saúde da população (MAKUCH DMV e ZAGONELI IPS, 2017).

O desenvolvimento desse novo sistema de assistência à saúde é fundamentado pela Constituição Federal de 1988. Nesse texto normativo é previsto, de forma ampla, os direitos à saúde, sendo tal direito positivado, de forma mais especifica, no artigo 196 da Constituição Federal, estabelecendo a responsabilidade objetiva do estado. O texto constitucional é fundamentado pelos princípios da universalidade, integralidade da assistência, preservação da autonomia, igualdade da assistência à saúde, direito a informação, com a participação da sociedade, melhorando o desenvolvimento das ações em saúde (BRASIL,1988).

Entretanto, mesmo com o processo de ensino focado nas políticas públicas de saúde, e seguindo as bases das diretrizes de ensino de enfermagem, ainda persistem dificuldades e fragilidades nos processos pedagógicos para abordar a saúde de forma ampliada, prejudicando o processo formativo do ensino, direcionada para atender as demandas sociais da população (PIRES AS, et al., 2014).

As instituições de ensino superior, vem sendo desafiadas a quebrar paradigmas, em relação a formação profissional, precisam propor ações inovadoras que reorientem as práticas de ensino, adotando estratégias que possam desenvolver profissionais críticos, reflexivos, humanísticos, transformadores da realidade social e agentes de mudanças, na perspectiva da reorganização das prática de saúde, com o intuito de contemplar as necessidades holísticos de forma individual e coletiva no âmbito do SUS (SOUZA KMJ, et al., 2017).

No estudo dos autores Peres CRFB, et al. (2018), no processo de ensino/aprendizagem em saúde, ainda perdura o modelo de atenção biologista, com conteúdos disciplinares fragmentados e ausência de diversificação dos cenários de aprendizagem, desintegração entre as disciplinas com o ensino/serviço, prejudicando o processo de ensino que se contrapõe as diretrizes curriculares de saúde, para formação de profissionais enfermeiros, despreparados para atuar no mercado. 
Assim, constata-se que precisa haver mudanças na abordagem pedagógica que alcance resultados efetivos na contemporaneidade, pois existem resistências na adoção de uma nova lógica de ensino, porque os docentes tiveram uma formação atrelada aos modelos tradicionais e tecnicistas, dificultando a adesão de novas tecnologias de ensino e a compreensão do processo de saúde e doença num contexto da integralidade. A adesão de novos saberes e habilidades é fundamental para mudar este contexto, contudo este processo de mudança já está em andamento com construções pedagógicas nas universidades (CRUZ et al., 2017).

Portanto, urge a necessidade de reorganizar o currículo, com abordagens dialógicas e inserindo as universidades no mundo do trabalho, com o propósito de tornar o ensino uma prática reflexiva, provocado pelas vivências nos cenários de saúde. Colocando o aluno como protagonista, juntamente com o professor, neste processo de aprendizagem (MAKUTH DMV e ZAGONEL IPS, 2017).

Para Chehuen Neto JA, et al. (2015), a PNSIPN tem o objetivo de garantir a equidade na atenção à saúde para esse segmento populacional, assegurando ações e serviços de saúde com justiça social, para as pessoas em condições de vulnerabilidades, com intuito de reduzir as desigualdades. A PNSIPN, foi aprovada, no dia 20 de novembro, que se comemora o dia nacional da consciência negra, pelo conselho nacional de saúde.

O Brasil, segundo Matos CC e Tourinho FS (2018), apresenta uma diminuição da qualidade e da expectativa de vida deste segmento, identificando maiores indicadores de mortalidade materna e infantil em decorrência da dificuldade de acesso aos serviços de saúde, e por viver em ambiente insalubres com predomínio de intensa violência, sobretudo entre jovens negros, quando comparados aos brancos.

O desconhecimento da PNSIPN por uma grande maioria da população negra 90,5\% reflete a dificuldade de comunicação das informações acerca dessa política, e falhas dos profissionais de saúde em suas competências no SUS, como também dos gestores dos serviços.

Segundo os autores os entrevistados negros souberam deste direito pelo entrevistador, se posicionando favorável a esta política pela insatisfação com o acesso e a qualidade dos serviços prestados na rede pública, contudo entrevistados afirmaram $(53,7 \%)$ que essa política reforça a discriminação racial, por achar que pode ser um privilégio, "pois somos todos iguais perante a lei, independente de raça" (CHEHUEN NETO JA, et al., 2015).

Verifica-se em nosso país a presença de desigualdades de acesso aos serviços de saúde, devido questões raciais, sociais, comprometendo a saúde integral da população negra, e com a instituição da portaria de $n^{\circ}$ 992, de 13 de maio de 2009, que sancionou a política nacional de saúde integral da população negra, foi uma medida para promover a equidade em saúde nestes povos (BRASIL, 2009).

E por esse motivo a PNSIPN, que aborda um dos princípios do SUS, a equidade, é relevante para que o discente possa conhecer e entender o processo dessa política, pois permitirá conhecer os principais agravos na população negra, o contexto de saúde local, as dificuldades e fragilidades vivenciadas diariamente da população, com garantia de acesso aos serviços do SUS, amenizando as iniquidades de saúde (INOCÊNCIO JF e TAVARES MM, 2014).

\section{CONCLUSÃO}

Ressalta-se, que na revisão sistemática, não foram encontrados artigos na formação do enfermeiro no cuidado da população negra indicando que os discentes e docentes precisam ter abordagens reflexivas da história de saúde dessa população.Os negros são a maioria que frequenta o SUS e que apresenta vulnerabilidades sociais, perfil epidemiológico elevado de adoecimento e com maior exposição a riscos sociais,ambientais e econômicos, com taxas elevadas de homicídios e criminalidade, com dificuldade de acesso nos serviços de saúde. Para tanto, a formação no curso de graduação de enfermagem deverá estar em consonância com os princípios do SUS e orientado pelas diretrizes curriculares, pois ainda apresenta fragilidades que deverão ser refletidas, para que o ensino no modelo tradicional e na visão biologicista, incorporem novos paradigmas e saberes com uma visão ampliada do processo saúde-doença. 


\section{REFERÊNCIAS}

1. BRASIL. Ministério da Educação. Resolução CNE/CES no 3, de 7 de novembro de 2001. Institui diretrizes curriculares nacionais do curso de graduação em enfermagem. Diário Oficial da União. 2001.

2. BRASIL. Ministério da Saúde. Política Nacional de Saúde Integral da População Negra: uma política para o SUS/Ministério da Saúde, Secretaria de Gestão Estratégica e Participativa, Departamento de Apoio à Gestão Participativa e ao Controle Social. 3nd ed. Brasília: Editora do Ministério da Saúde, 2017; 44 p.

3. BRASIL. Portaria oํ 992, de 13 de maio de 2009. Institui a Política Nacional de Saúde Integral da População Negra. Diário Oficial da União. 2009.

4. BRASIL. Senado Federal. Constituição Federal de 1988, 1988.

5. BRASIL. Senado Federal. Constituição Federal de 1988. 1988.

6. CHEHUEN NETO JÁ, et al. Política Nacional de Saúde Integral da População Negra: implementação, conhecimento e aspectos socioeconômicos sob a perspectiva desse segmento populacional. Ciencia \& saude coletiva, 2015; 20: 1909-1916.

7. CRUZ RAO, et al. Reflexões à luz da Teoria da Complexidade e a formação do enfermeiro. Revista Brasileira de Enfermagem, 2017; 70(1): 236-239.

8. GANASSIN FMH. Avaliação do processo de implementação de mudança curricular de cursos de enfermagem: um estudo em duas universidades públicas do estado de Mato Grosso do Sul. Tese (doutorado) - Universidade Estadual de Campinas, Faculdade de Educação, Campinas, São Paulo, 2015; 254 p.

9. GAZZONI F, et al. O papel das IES no desenvolvimento sustentável: estudo de caso da Universidade Federal de Santa Maria. Revista Gestão Universitária na América Latina-GUAL, 2018; 11(1): 48-70.

10. INOCÉNCIO JF, TAVARES MM. A percepção do enfermeiro quanto à saúde do negro. Revista PróUniverSUS, 2013; 4(2), 01-01.

11. LAGES SRC, et al. O preconceito racial como determinante social da saúde-a invisibilidade da anemia falciforme. Gerais: Revista Interinstitucional de Psicologia, 2017; 10(1): 109-122.

12. MAGALHÃES SMF, et al. Educação em Enfermagem: conceituando projeto pedagógico na visão de professores. Acta Paulista de Enfermagem, 2017; 30(3): 247-253.

13. MAKUCH DMV, ZAGONEL IPS. Abordagem pedagógica na implementação de programas curriculares na formação do enfermeiro. Escola Anna Nery Revista de Enfermagem, 2017; 21(4): 1-9.

14. MATOS CC, TOURINHO FS. Saúde da População Negra: percepção de residentes e preceptores de Saúde da Família e Medicina de Família e Comunidade. Revista Brasileira de Medicina Família e Comunidade, 2018; 13(40): 1-12.

15. MENDES VS, et al. Racismo Biológico e suas implicações no ensinar-cuidar a saúde da população negra. Revista da ABPN, 2015; 7(16):190-213.

16. MOHER D, et al. Preferred reporting items for systematic review and meta-analysis protocols (PRISMA-P) 2015 statement. Syst Rev, 2015; 4 (1).

17. PERES $\mathrm{C}$, et al. A dialectical view of curriculum changes in nursing training. Revista da Escola de Enfermagem da USP, 2018; 52: e03397.

18. PIRES AS, et al. A formação de enfermagem na graduação: uma revisão integrativa da literatura. Rev. Enferm. UERJ, 2014; 22(5): 705-711.

19. PORTO A. O sistema de saúde do escravo no Brasil do século XIX: doenças, instituições e práticas terapêuticas. História, Ciências, Saúde-Manguinhos, 2006; 13(4).

20. SANTANA RAR, et al. A equidade racial e a educação das relações étnico-raciais nos cursos de Saúde. Interface Comunicação, Saúde, Educação, 2019; 23, ISSN 1807-5762.

21. SOUZA KMJD, et al. Contribuições da saúde coletiva para o trabalho de enfermeiros. Revista Brasileira de Enfermagem, 2017; 70(3); 543-549.

22. WINTERS JR, et al. A formação em enfermagem orientada aos princípios do Sistema Único de Saúde: percepção dos formandos. Escola Anna Nery Revista de Enfermagem, 2016; 20(2): 248-253. 